\title{
Editorial \\ Recent advances in systemic therapy for breast cancer: new technologies for a new era
}

\author{
Paul Ellis
}

Department of Medical Oncology, Guy's Hospital, London SE19RT, UK

Corresponding author: Paul Ellis, paul.ellis@gstt.nhs.uk

Published: 28 August 2009

Breast Cancer Research 2009, 11:107 (doi:10.1186/bcr2254)

This article is online at http://breast-cancer-research.com/content/11/4/107

(c) 2009 BioMed Central Ltd

See related reviews series, http://breast-cancer-research.com/series/bcr_systemic_therapy

The past decade has brought enormous advances in the way we manage breast cancer patients with systemic therapies. An explosion in our understanding of the molecular pathogenesis of breast cancer, a harnessing of powerful new diagnostic technologies, and the culmination of a series of pivotal randomized trials of cytotoxic and novel targeted therapies have yielded significant improvements in outcomes for patients with both early and advanced disease. A series of review articles published in Breast Cancer Research written by leading clinical researchers explores these advances in different areas of breast cancer management.

Miles [1] discusses new advances in the management of metastatic breast cancer focusing particularly on human epidermal growth factor receptor (HER)-negative disease. His review addresses pivotal trials of cytotoxic therapy and highlights optimal management in 2009. In addition, it focuses on the role played by targeted agents, and particularly antiangiogenic agents, highlighting new data with agents such as bevacizumab. New approaches to the management of 'triple negative' breast cancer are also discussed.

Advances in the management of patients with HER2-positive metastatic breast cancer are reviewed by Esteva and colleagues [2]. Optimal use of currently available targeted agents such as trastuzumab and lapatanib are discussed, including the use of both in clinical scenarios such as relapse after adjuvant trastuzumab or progression following first-line use of trastuzumab in the metastatic setting. The potential role of newer agents targeting the HER family are also highlighted, as is the case for the use of combined targeted agents.

Key issues in the use of adjuvant systemic therapy for early breast cancer are reviewed by Martín and López-Tarruella [3]. Current 'gold standard' options for the use of adjuvant chemotherapy are discussed within the setting of nodepositive and node-negative disease. Issues such as which patients should receive taxane-based chemotherapy and the optimal schedule for such regimens are covered. Clinical trials of novel targeted therapies are also discussed.

The increasing use of neoadjuvant systemic therapy is highlighted by Untch and von Minckwitz [4]. Pivotal trials in this area are reviewed, and the use of clinical and biological surrogate markers of long-term outcome is discussed.

Finally, Di Leo and colleagues [5] discuss the evidence base behind some of the new molecular prognostic and predictive profile technologies. They review the concept of using biomarkers to predict outcome and the place of tests such as Oncotype DX (Genomic Health, Redwood, CA, USA) and MammaPrint (Agendia, Huntington Beach, CA, USA). What do they add over existing expertise and in which areas should they be used in everyday practice, if at all? They also discuss ongoing clinical trials using these technologies, such as the TAILORx (Trial for Assigning IndividuaLized Options for Treatment) and MINDACT (Microarray In Node-negative Disease may Avoid ChemoTherapy) trials.

In summary these reviews provide a snapshot of current state-of-the-art medical practice in the use of systemic cytotoxic and targeted therapy for breast cancer management. If the past decade has helped to crystallize incremental gains in outcome for patients with early and metastatic disease, this decade promises further advance by using the molecular advances described above to help identify which groups of patients may gain from particular types of therapy.

\section{Competing interests}

The author declares that they have no competing interests.

HER = human epidermal growth factor receptor. 


\section{References}

1. Miles DW: Recent advances in systemic therapy. When HER2 is not the target: advances in the treatment of HER2-negative metastatic breast cancer. Breast Cancer Res 2009, 11:208.

2. Morrow PKH, Zambrana F, Esteva FJ: Recent advances in systemic therapy. Advances in systemic therapy for HER2 positive metastatic breast cancer. Breast Cancer Res 2009, 11: 207.

3. López-Tarruella S, Martín M: Recent advances in systemic therapy. Advances in adjuvant systemic chemotherapy of early breast cancer. Breast Cancer Res 2009, 11:204.

4. Untch $M$, von Minckwitz G: Recent advances in systemic therapy. Advances in neoadjuvant (primary) systemic therapy with cytotoxic agents. Breast Cancer Res 2009, 11:203.

5. Oakman C, Bessi S, Zafarana E, Galardi F, Biganzoli L, Di Leo A: Recent advances in systemic therapy. New diagnostics and biological predictors of outcome in early breast cancer. Breast Cancer Res 2009, 11:205. 